\title{
Analysis of Material Behavior for Friction in a Nozzle for Turbomachinery and High Speed Vehicles
}

\author{
S. M. Prabhu ${ }^{1 *}$, Abbas Mohadeen ${ }^{2}$ \\ ${ }^{1}$ NIMS of Engineering \& Technology, Jaipur, India; ${ }^{2}$ Mamallan Institute of Technology, Sriperumbudur, India. \\ Email: "psmallapur@hotmail.com,prabhusm2005@gmail.com
}

Received January $17^{\text {th }}, 2011$; revised April $6^{\text {th }}, 2011$; accepted July $20^{\text {th }}, 2011$.

\begin{abstract}
Shock-induced separation of turbulent boundary layers represents a long-studied problem in compressible flow, bearing, for example, on applications in high speed aerodynamics, rocketry, wind tunnel design, and turbomachinery. Experimental investigations have generally sought to expose essential physics using geometrically simple configurations.
\end{abstract}

Keywords: Adiabatic Proces, Friction Efects, Nozzle Modeling, Materialbehavior

\section{Introduction}

Shock-induced separation of turbulent boundary layers represents a long-studied problem in compressible flow, bearing, for example, on applications in high speed aerodynamics, rocketry, wind tunnel design, and turbo machinery. Experimental investigations have generally sought to expose essential physics using geometrically simple configurations, e.g., supersonic flow over compression ramps [1-4], curved surfaces [2], backward and forward facing steps [2], simplified wing shapes [5], and various blunt objects $[4,6,7]$. While a variety of computational and analytical methods have also been developed for treating the problem, the methods are typically applicable to specific compressible flow regimes, i.e., transonic, supersonic or hypersonic flow, and moreover, due to the intrinsic unsteadiness of the separation process, require problem-specific tuning.

The rocket nozzle can surely be described as the epitome of elegant simplicity. The primary function of a nozzle is to channel and accelerate the combustion products produced by the burning propellant in such as way as to maximize the velocity of the exhaust at the exit, to supersonic velocity. The familiar rocket nozzle, also known as a convergent-divergent, or de Laval nozzle, accomplishes this remarkable feat by simple geometry. In other words, it does this by varying the cross-sectional area (or diameter) in an exacting form. The analysis of a rocket nozzle involves the concept of "steady, one-di- mensional compressible fluid flow of an ideal gas". Briefly, this means that:

1) The flow of the fluid (exhaust gases + condensed particles) is constant and does not change over time during the burn.

2) One-dimensional flow means that the direction of the flow is along a straight line. For a nozzle, the flow is assumed to be along the axis of symmetry.

3) The flow is compressible. The concept of compressible fluid flow is usually employed for gases moving at high (usually supersonic) velocity, unlike the concept of incompressible flow, which is used for liquids and gases moving at a speed well below sonic velocity. A compressible fluid exhibits significant changes in density, an incompressible fluid does not.

4) The concept of an ideal gas is a simplifying assumption, one that allows use of a direct relationship between pressure, density and temperature, which are properties that are particularly important in analyzing flow through a nozzle.

Fluid properties, such as velocity, density, pressure and temperature, in compressible fluid flow, are affected by

1) Cross-sectional area change.

2) Friction.

3) Heat loss to the surroundings.

\section{Experimental Setup}

Figure 1 shows the Experimental setup of Nozzle under 
adabatic flow conditions. The heat flux distribution is determined by thermocouple connected on the work piece at definite distances. Cu-Ni Thermocouples were attached on the surface of the work piece. The flame was generated by acetylene and oxygen gasses as in gas welding process. The flame was kept at distances from 5 $\mathrm{cm}$ to $20 \mathrm{~cm}$ and the heat flux distribution studied. During a typical experimental run the powers were varied to achieve different base late temperature and hence Rayleigh numbers. Due to temperature constraints the parameters of the heat input were restricted to maximum base plate temperature of $250^{\circ} \mathrm{C}$.

\section{Model Proposal}

This paper investigates time-average, shock-induced turbulent boundary layer separation in over-expanded rocket nozzles. Although focused on this particular problem, much of the development applies to the same broad family of shock-separated flows encompassed by the free interaction model. The objectives are to first present and examine an alternative to the free interaction model, with a view toward obtaining a fuller understanding of the free interaction process. Simple scale analyses of transverse momentum transport across the separating boundary layer are presented and used to derive criteria for estimating the approximate time-average separation pressure ratio, $P_{i} / P_{p}$, as a function of the in viscid separation Mach Number, $M_{i}=M\left(x_{i}\right)$, where $P_{i}$ is the time-average wall pressure at the point of incipient separation, $x_{i}$, and $P_{p}$ is the peak wall pressure at the downstream limit of the shock interaction zone, $x_{p}$; see Figure 1. In the case of rocket nozzle flows, where separation-induced side loading constitutes an intrinsic feature of low altitude flight $[15,16]$, knowledge of the separation criterion $P_{i}$

$$
\rho_{0}=F_{0}(M)
$$

is crucial since it allows determination of the corresponding separation line location. The time average pressure gradient over the shock interaction zone $\left(x_{i^{-}}\right.$ $x-x_{p}$ ), given approximately by

$$
\frac{\partial p}{\partial x}=\frac{\partial_{0}-\partial_{i}}{\rho_{i}}
$$

in reality reflects the intermittent, random motion of the shock between $x i$ and $x_{p}$ [3]. As the shock-compression wave system oscillates randomly above (and partially within) the boundary layer, the associated pressure jump across the system is transmitted across the boundary layer on a time scale $\left(x-\delta_{i}\right) / \sqrt{k R T_{i}}$, where $\delta_{i}$ and $T_{i}$ are the characteristic boundary layer thickness and temperature in the vicinity of $x_{i}$. Under typical experimental conditions, $T_{s}$ is much shorter than the slow time scale, $f=1 \mathrm{~s}$ (where $T_{s} \approx 1$ to $10 \mu \mathrm{s}$ ); thus, the instantaneous separa-

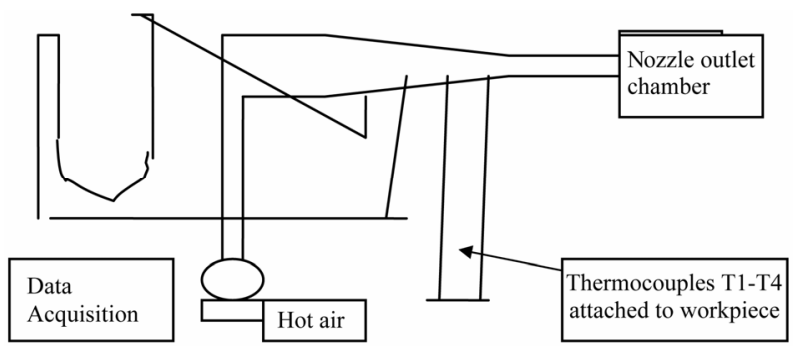

(a)

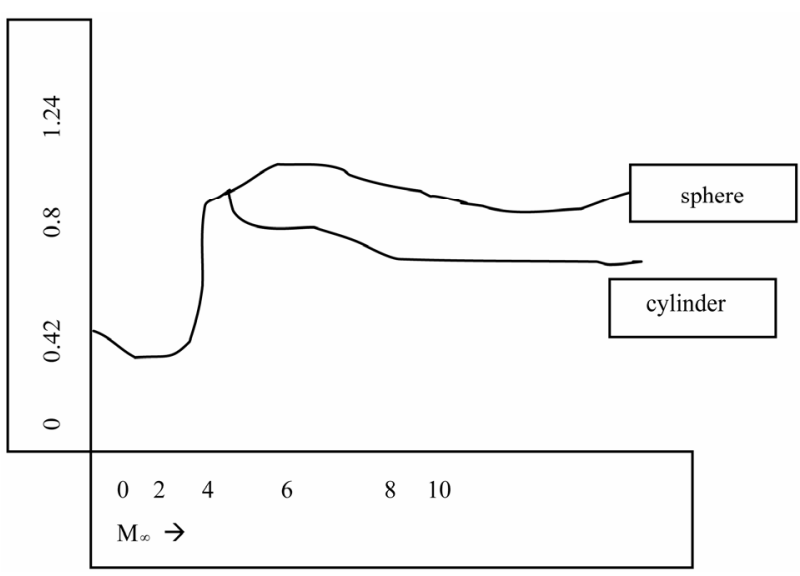

(b)

Figure 1. (a) Experimental setup of Nozzle under adabatic flow conditions; (b) drag coefficient for sphere and cylinder in hypersonic flow.

tion point essentially tracks the random position of the shock-compression wave system, where the position of the separation point is described by a Gaussian distribution over the length of the interaction zone [3].

\section{Scale Analysis I}

Considering the vertical momentum balance immediately downstream of the separation point $x_{s}$, it is recognized that the boundary layer lifts off of the wall due to a vertical gradient in pressure $[2,30]$. Thus, the vertical advection of vertical momentum must be of the order of the vertical pressure gradient:

$$
\frac{\rho_{v} \partial v}{\partial y}=\frac{\partial p}{\partial y}
$$

or in approximate form,

$$
\frac{\rho \partial v}{y}=-\partial v / \partial y
$$

where the density $\rho_{2}$ of the boundary layer near $x_{s}$ is approximated as the free stream density downstream of the shock, $v s$ is the characteristic vertical velocity component within the boundary layer near $x_{s}$, and $\delta_{s} \approx \delta_{i}$ is the characteristic boundary layer thickness near $x_{s} P_{p}-P_{2}$, is estimated as the difference between the peak wall pressure, 
$P_{p}$, in the vicinity of $x_{p}$ and the free stream pressure immediately downstream of the oblique shock. The density estimate in (4) recognizes that the boundary layer has passed through the compression wave system at the foot of the oblique shock.

Eliminating $\delta_{s}$ from (4) and solving for $v_{s}$ yields

$$
\frac{v_{s}}{€}=\left(v_{p}-\rho_{1}\right) / \rho_{1}
$$

From Figure 2, we note that at the separation point, $v_{s}$ is related to the characteristic horizontal velocity component, $u_{s}$ by

$$
\frac{v_{1}}{u_{1}}=\tan \theta,
$$

where $\theta$ is the characteristic angle of deflection between the separating boundary layer and the nozzle wall. The magnitude of $u_{s}$ is estimated by again noting that the boundary layer flow has passed through the compression wave system at the foot of the oblique shock and that, as indicated in Figure 2, the time average turbulent boundary layer velocity profile is nearly flat. Thus, $u_{s}$ is on the order of the $x$-component $\left(U_{2}\right)$ of the inviscid flow velocity $\left(v_{2}\right)$ immediately downstream of the oblique shock:

$$
u_{x}=u_{2-v_{2}} \cos \theta
$$

Using the ideal gas relation, $\rho_{2}=k P_{2} /\left(k R T_{2}\right)$, and inserting (5) in (6) we then obtain

$$
\tan \theta=\frac{1}{M_{2 x}}\left(\frac{\rho_{p}-\rho_{2}}{\rho_{2} k}\right)^{0.5}
$$

where $M_{2 x}=M_{2} \cos \theta$, and $M_{2}$ is the in viscid flow Mach Number immediately downstream of the oblique shock.

Rewriting $P_{p} / P_{2}$ as $\left(P_{p} / P_{1}\right)\left(P_{1} / P_{2}\right)$ and solving (8) for $P_{p} / P_{1}$ finally yields

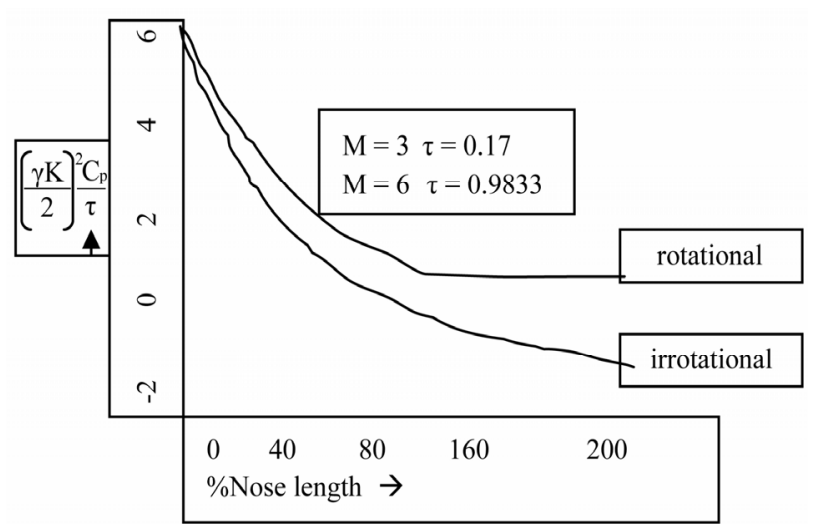

Figure 2. Plot of nose length vs friction adiabatic coefficient of the nozzle.

$$
\frac{\rho_{1}}{\rho_{p}}=\frac{1}{1+\sin ^{2} \theta k M_{2}^{2}} \frac{P_{1}}{p_{2}}
$$

Identifying $P_{1} / P_{p}$ as the critical wall pressure ratio at which separation occurs, i.e., $P_{1} / P_{p} \approx P_{i} / P_{p}$, noting that $M_{2}$ is given by the oblique shock relation

$$
M_{2}^{2}=\frac{(k-1) M_{2}{ }^{2} \sin ^{2} \beta+(2 k-1)}{2 k M_{y}{ }^{2} \sin ^{2} \beta_{2}+(2 k-1)}
$$

and recognizing that $M_{i} \approx M_{1}$, where $M_{1}$ is the free stream Mach Number immediately upstream of the oblique shock, it is seen that (9) provides an explicit, physicallybased relationship between $P_{i} / P_{p}$ and the pressure ratio, $P_{1} / P_{2}$, across the oblique shock. The next scale analysis refines the estimates for stream wise inertia and crosslayer pressure gradient, and leads to a near-identity between $P_{i} / P_{p}$ and $P_{1} / P_{2}$.

\subsection{Optimization of Contour Nozzle Design Including Viscous Effects}

Here we present a genetic algorithm for design of Mach 12 contoured Nozzle. The objective is to produce a uniform velocity (Mach Number) over the entrance region in the nozzle exit plane and to limit the flow angularity at the exit plane, over the same region within a given limit. At hypersonic wind tunnel nozzles with Mach Numbers greater than 8 are dominated with strong viscous effects, the nozzle contour generated by conventional method of characteristics does not meet the design requirements when boundary layer corrections are made. In the present work parallel Aerodynamic simulator code (PARAS) or FLUENT. The former uses the surface oriented mesh system to simulate the flow inside the axisymmetric nozzle. The code solves Navier stokes equations using a Finite volume approach and is convenient and fast. To represent the nozzle contour generated by conventional method of characteristics. CFD solutions are used to evaluate the objective function in each function evaluation of the Genetic Algorithm (GA) process. To represent the nozzle contour in terms of certain parameter vector $\mathbf{P}\left(p_{1}, p_{2}, \cdots, p_{n}\right)$ the nozzle contour is divided into 5 segments and is represented as follows.:

$$
\begin{array}{rlrl}
\text { For } 0 \leq<x \leq x_{1} & r=a_{0}+a_{1} x+a_{2} x^{2}+a_{3} x^{3} \\
x_{1} \leq<x \leq x_{2} & r=a_{0}+a_{1} x+a_{2} x^{2}+a_{3} x^{3} \\
x_{2} \leq<x \leq x_{3} & r=a_{0}+a_{1} x+a_{2} x^{2}+a_{3} x^{3} \\
x_{3} \leq<x \leq x_{4} & r=a_{0}+a_{1} x+a_{2} x^{2}+a_{3} x^{3} \\
x_{4} \leq<x \leq x_{5} & r=a_{0}+a_{1} x+a_{2} x^{2}+a_{3} x^{3}
\end{array}
$$

The above five cubic spheres result in 20 coefficients $\left(a_{0}, a_{1}, \cdots, a_{20}\right)$ assuming that the locations $x_{1}, x_{2}, \cdots, x_{5}$ are known. The slopes at the nozzle throat and the exit plane are assumed to be zero. The continuity of radius, slope and curvature at the four interfaces give 4 condi- 
tions. With these 20 conditions, the above set of linear simultaneous equations is solved using the Gauss Siedel method. After getting the coefficients $a_{0}, \cdots, a_{19}$ the nozzle contour can be determined. The length of the nozzle is also kept as a floating parmeter. A constant inlet Mach Number of unity has been taken and the tunnel operating conditions have been taken as $p_{0}=68 \mathrm{KSC}, T_{0}=1500 \mathrm{~K}$ and ratio of specific heats $\gamma=1.31$. Besides the radii $r$ th, $r_{1}, r_{2}, r_{3}$, and $r_{4}$ the length of the nozzle $L\left(=x_{5}\right)$ is also considered as a parameter. The nozzle exit radius is 0.5 $\mathrm{m}$ which is the test section radius is $0.5 \mathrm{~m}$ which is the test section radius, and is fixed. The nominal values of these parameters are arrived at using the MOC contour with the boundary layer correction.

The objective function consists of 2 parts. First part takes into account the deviation of actual Mach Number $M_{\text {act }}$ from the target Mach Number $M_{\text {tar }}(=12)$ and the second part of the deviation of flow angularity $\alpha_{\text {act }}$ from the target flow angularity $\alpha_{\text {tar }}(0.2 \mathrm{deg})$. These are evaluated at the core of the nozzle exit. Thus we have $\left(M_{\mathrm{tar}}-\right.$ Mach at target)

$$
\operatorname{Obj}(P)=-1 / N\left[\phi_{M} \sum\left(M_{\text {act }}-M_{\text {tar }}\right)^{2}+\phi_{\alpha} \sum\left(\alpha_{\text {act }}-\alpha_{\text {tar }}\right)^{2}\right]
$$

where $\mathbf{P}$ is the parameter vector and $\mathbf{N}$ is number of grid cells in the core of the nozzle exit. Values of $\phi_{M}$ and $\phi_{\alpha}$ are taken as 0.7 and 0.28 respectively, which are the weighting factors.

Figure 7 shows the Objective function as defined above with number of generations. It is seen that the objective function falls by $30 \%$ in about 68 generations.

\subsection{Modeling of Hypersonic Flows}

The Navier Stokes equations for the hypersonic flow are given by the continuity and momentum equations.

Continuity Equation

$$
\begin{gathered}
\frac{\partial u}{\partial x}+\frac{\partial v}{\partial y}=0 \\
u \frac{\partial v}{\partial x}+v \frac{\partial v}{\partial y}=-\frac{g \beta k}{v} \frac{\partial T}{\partial x}
\end{gathered}
$$

where volumetric heat sources $(x, y, z)$ represents the contribution of frictional heating. The parameters $\rho C_{p} \&$ $k_{e}$ may depend on $y \& z$ but remain independent of $x$. More importantly the contributing of axial conduction deferred to the subsequent is neglected, hence Equation (4) reduces

$$
\frac{u \partial T}{\partial x}+\frac{v \partial T}{\partial x}=\alpha\left(\frac{\partial^{2} T}{\partial x^{2}}+\frac{\partial^{2} T}{\partial y^{2}}\right)+\frac{\mu}{K \rho C p}\left(u^{2}+v^{2}\right)=0
$$

When these are non dimensionalized $g$ the following definitions of variables

$$
\begin{aligned}
& x^{*}=x / l, y^{*}=y / l, z^{*}=z / l, u^{*}=u / V_{\mathrm{inf}}, \\
& P^{*}=P / \rho_{\infty} v_{\infty}^{2}, \rho^{*}=\rho / \rho_{\infty}
\end{aligned}
$$

we get

$$
\begin{gathered}
\nabla \rho^{*} u^{*}=0 \\
\rho^{*} \nabla \cdot \nabla u=0^{* *} \\
u \nabla \frac{p^{*}}{\rho^{*}}=0
\end{gathered}
$$

For flow tangent to body $V \cdot n=0$.

Let $n_{x}, n_{y}, n_{z}$ be the vector. Using the final pressure ratio equations can be derived for the nozzle as below. Consider the hyperonic flow over a given body in the limit of large $M_{\infty}$. The flow is again governed by Equations (4)-(6) we get the Bcondns as

$$
\begin{gathered}
p_{2} \rightarrow \sin ^{2} \beta(\gamma \rightarrow) \gamma \\
\rho_{2} \rightarrow \frac{\gamma+1}{\gamma-1} \\
u_{2} \rightarrow 1-\left(2 \sin ^{2} \beta(\gamma-1)\right) \\
v_{2} \rightarrow \sin ^{2} \beta(\gamma-1)
\end{gathered}
$$

From this consideration we can seethe Mach Number is independent and this follows from the governing Equations of motion with the appropriate BCs. Written in limit of high Mach Number. Hence when the free stream Mach Number is very high the dimensionless variables become independent on Mach Number, this trend applies to any quantities derived from these dimensionless variables. The drag coefficient follows a plot as in Figure 1 below.

Examining the governing flow Equation upon which hyperbolic similarity is based (Equations of continuity \& motion) in dimensionless form)The similarity principle holds for both irrotational and rotational flow as shown in Figure 2 where the two curves for irrotational \& rotational flow overlap each other. The surface pressure distribution is shown in Figure below.

The sphere drag achieve Mach no independence at lower Ma. For blunt cone end ogive cylinders the velocity distributions is given below.

The maximum location of the shock is btaies as shown in Figures 4 and 5.

The main point in this discussion however, to find the farthest shock location downstream. Figure 5 shows the possible as function of retreat of the location of the shock wave from the maximum location. When the entrance Mach Number is infinity, $P_{2}$, if the shock location is at the Maximum length, than shock at $M_{2}<1$ results in 
$4 f L / D=0.3$ and possible.

The proposed procedure is based on Figure 5 .

1) Calculated the extra $4 f L / D$ and subtract the actual extra $4 f L / D$ assuming shock at the left side (at the max length).

2) Calculated the extra $4 f L / D$ and subtract the actual extra $4 f L / D D$ assuming shock at the right side (at the entrance).

3) According to the positive or negative utilizes your root finding procedure.

From numerical point of view, the Mach Number equal infinity when left side assume result in infinity length of possible extra (the whole flow in the tube is subsonic). To overcome this numerical problem it is suggested to start the calculation from distance from the right hand side.

Let us denote

$$
4 f L / D>4 f L_{\max } / D \sim 0.34
$$

Note that $4 f L / D<4 f L / D_{\max }$ is smaller than $P_{2}$. The requirement that has to satisfied is that denote as difference between the maximum possible of length in which the flow supersonic achieved and the actual length in which the flow is supersonic as in Figure 8. The retreating length is expressed as subsonic but as the Figure 8 shows the entrance Mach Number, $M_{1}$ is reduced after the maximum length is exceeded. From numerical point of view, the Mach Number equal infinity when left side assume result in infinity length of possible extra (the whole flow in the tube is subsonic). To overcome this numerical problem it is suggested to start the calculation from distance from the right hand side.

\section{Results and Discussion}

Figure 2 shows the plot of Nose length vs friction adiabatic coefficient of the nozzle Figure 3 gives the Pressure distributions for ogive cylinders: illustration of hypersonic similarity a) $K=0.5$ b) $K=1.0$. Figure 4 shows the Surface pressure distribution at $x / R_{n}=8 \alpha=20^{*}, \gamma=$ 1.67, $R e=86000, \theta_{c}=15$. Figure 5 Pressure distribution over blunt nosed cone, compared with pointed cone. Figure 6 shows the Fanno flow characteristics.

Figure 7 depicts the Mach Number variation with friction headloss. Figure 8 gives the depiction of pressure drop variation with friction headloss. At hypersonic wind tunnel nozzles with Mach Numbers greater than 8 are dominated with strong viscous effects, the nozzle contour generated by conventional method of characteristics does not meet the design requirements when boundary layer corrections are made. In the present work parallel aerodynamic simulator code (PARAS) or FLUENT. The requirement that has to satisfied is that denote as difference between the maximum possible of length in which the flow supersonic achieved and the actual length in which

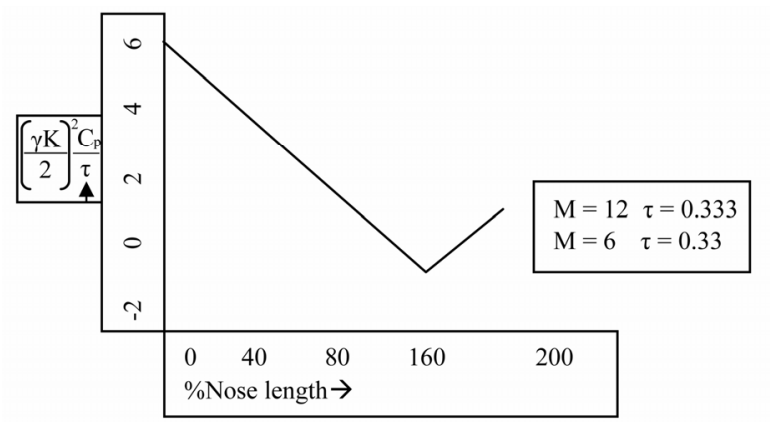

Figure 3. Pressure distributions for ogive cylinders: illustration of hypersonic similarity (a) $K=0.5$ (b) $K=1.0$.

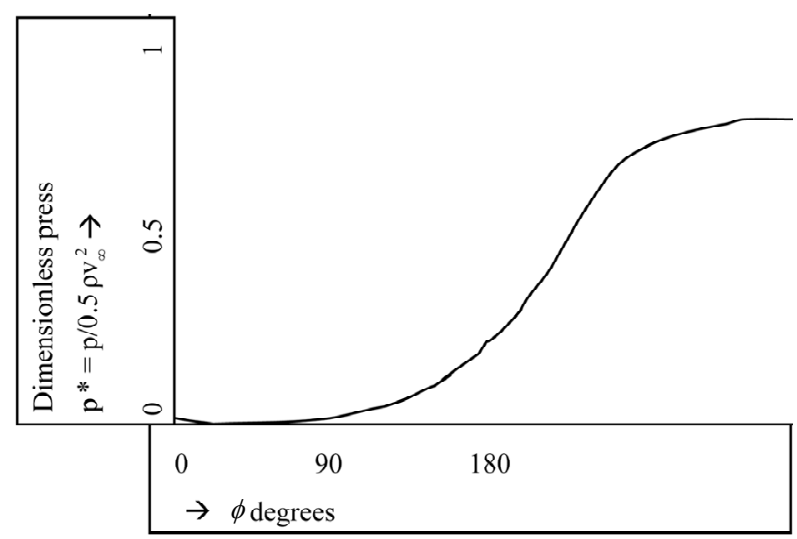

Figure 4. Surface pressure distribution at $x / R_{n}=8, \alpha=20$, $\gamma=1.67, R e=86000, \theta_{c}=15$.

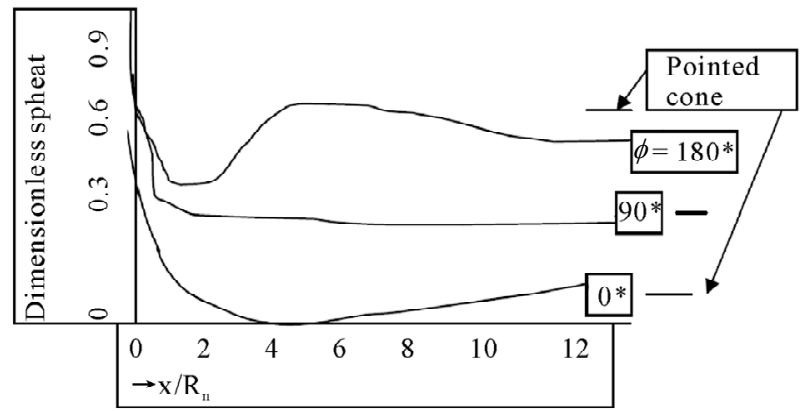

Figure 5. Pressure distribution over blunt nosed cone, compared with pointed cone.

the flow is supersonic as in Figure 8. From numerical point of view, the Mach Number equal infinity when left side assume result in infinity length of possible extra (the whole flow in the tube is subsonic).

\section{Conclusions and Suggestions}

Time-average, shock-induced, turbulent boundary layer separation has been investigated using a combination of heuristics, simple analytical models, and experiments, with a focus on separation in over expanded rocket nozzles. Two simple scaling analyses are presented in which 


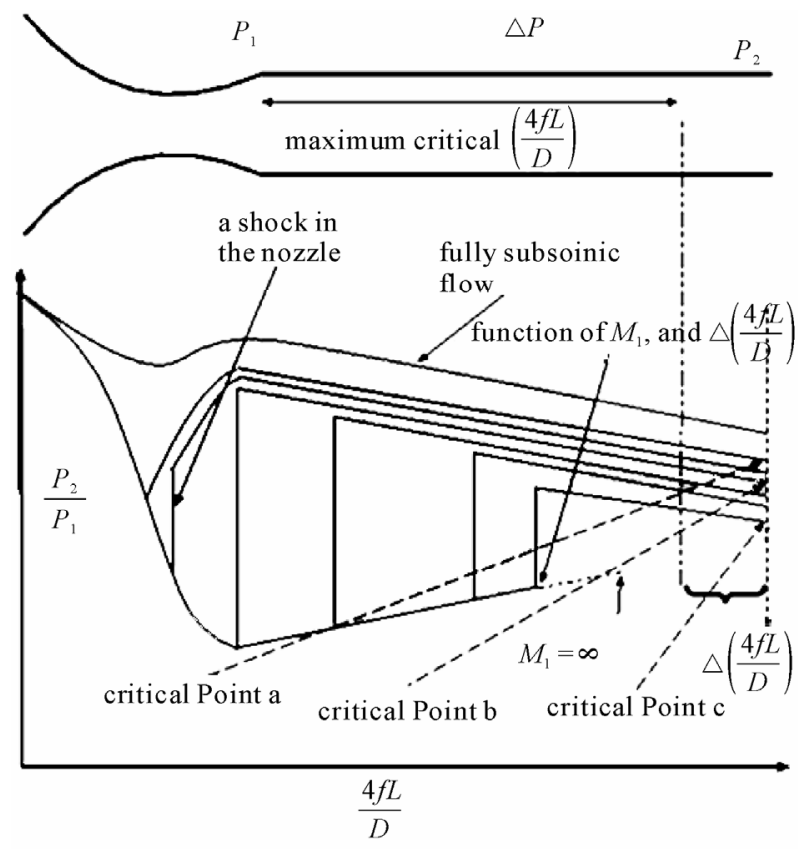

Figure 6. Fanno flow characteristics.

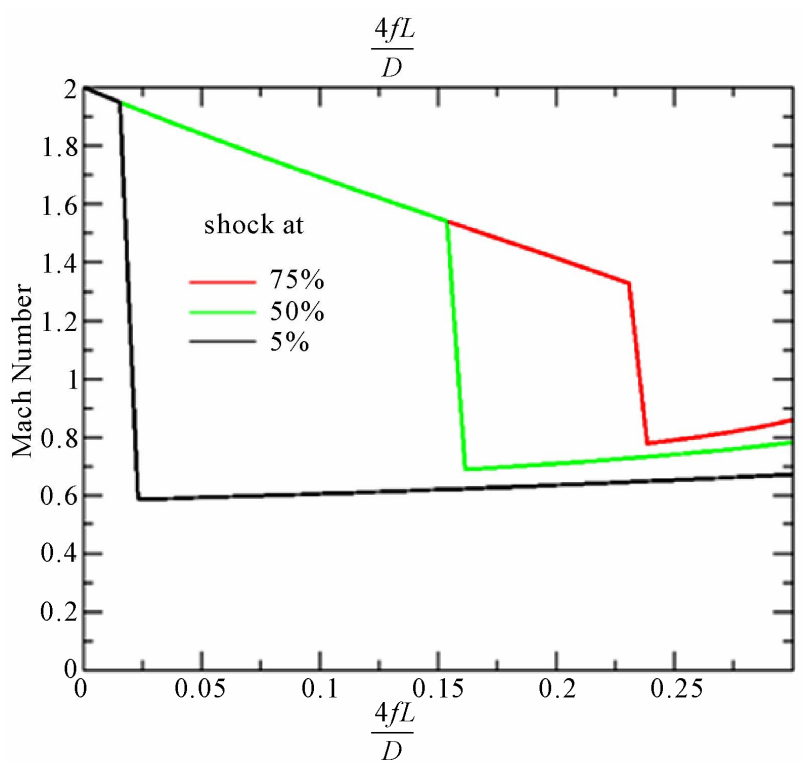

Figure 7. Mach Number variation with friction head loss.

separation is viewed as reflecting a balance between streamwise boundary layer inertia and the cross-layer pressure gradient. These lead to two theoretical expressions for the time average separation pressure ratio, stated as a function of the inviscid Mach Number at the point of incipient separation. In both models, explicit relationships between the separation pressure ratio (at the wall) and the classical oblique shock ratio are obtained; the second model, representing a refinement of the first, demonstrates for the first time that the separation pres-

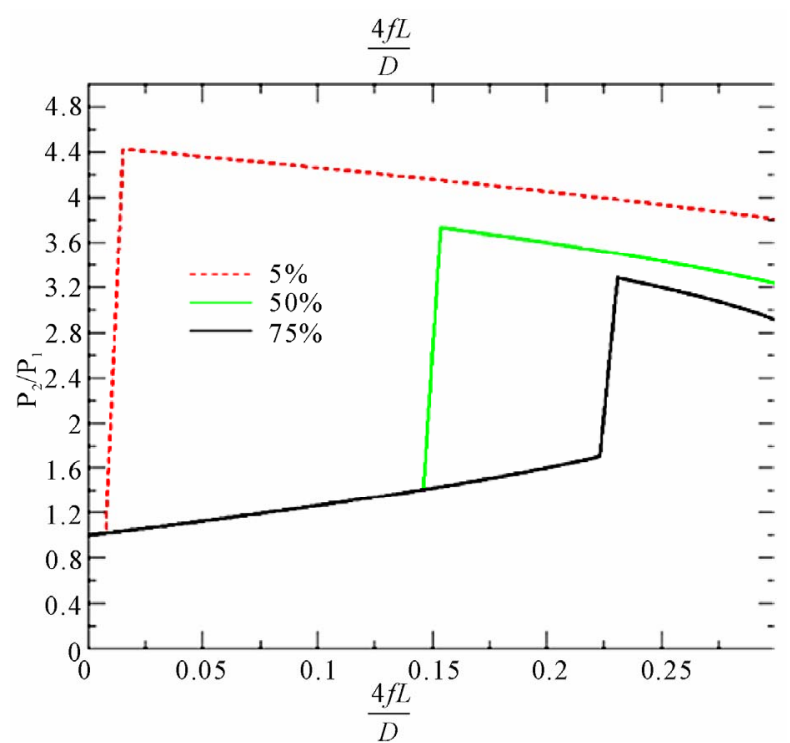

Figure 8. Plot of Pressure ratio in the nozzle to aspect ratio of nozzle.

sure ratio is, to a good approximation, determined by the oblique shock pressure ratio.

\section{REFERENCES}

[1] G. Settles, "Details of a Shock-Separated Turbulent Boundary Layer at a Compression Corner," AIAA Journal, Vol. 14, No. 12, 1976, pp. 1709-1715. doi:10.2514/3.61513

[2] D. R. Chapman, D. M. Kuehn and H. K. Larson, "Investigation of Separated Flows in Supersonic and Subsonic Streams with Emphasis on the Effect of Transition," NACA Report 1356, 1958.

[3] M. E. Erengil and D. S. Dolling, "Correlation of Separation Shock Motion with Pressure Fluctuations in the Incoming Boundary Layer," AIAA Journal, Vol. 29, No. 11, 1990, pp. 1868-1877. doi:10.2514/3.10812

[4] D. S. Dolling and L. Brusniak, "Separation Shock Motion in Fin, Cylinder, and Compression Ramp-Induced Turbulent Interactions," AIAA Journal, Vol. 27, No. 6, 1988, pp. 734-742. doi: $10.2514 / 3.10173$

[5] J. Ackeret, F. Feldman and N. Rott, "Investigations of Compression Shocks and Boundary Layers in Gases Moving at High Speed," NACA TM No. 1113, 1947.

[6] D. S. Dolling and D. R. Smith, "Separation Shock Dynamics in Mach 5 Turbulent Interactions Induced by Cylinders," AIAA Journal, Vol. 27, No. 12, 1989, pp. 1698-1706. doi: $10.2514 / 3.10323$

[7] R. A. Gramann and D. S. Dolling, "Detection of Turbulent Boundary-Layer Separation Using Fluctuating Wall Pressure Signals," AIAA Journal, Vol. 28, No. 6, 1989, pp. 1052-1056. doi: $10.2514 / 3.25164$

[8] J. Ostlund, "Flow Processes in Rocket Engine Nozzles with Focus on Flow-Separation and Side-Loads," Licentiate Thesis, Royal Inst. of Technology, Stockholm, TRITA-MEK, Technique Report, 2002, p. 1112. 